\title{
Positive Feedback Loop of Long Noncoding RNA OASL-IT1 and Innate Immune Response Restricts the Replication of Zika Virus in Epithelial A549 Cells
}

\author{
Yi Wang ${ }^{a, b}$ Zhiting Huo ${ }^{a, b}$ Quanshi Lin ${ }^{a, b}$ Yuxia Lin ${ }^{a, b}$ Cancan Chen ${ }^{c}$ \\ Yanxia Huang ${ }^{a, b}$ Changbai Huang ${ }^{a, b}$ Junsong Zhang ${ }^{d}$ Junfang $\mathrm{He}^{a, b}$ \\ Chao Liu ${ }^{a, e}$ Ping Zhang ${ }^{a}$, b
}

\begin{abstract}
aKey Laboratory of Tropical Disease Control, Ministry of Education, Zhongshan School of Medicine, Sun Yat-sen University, Guangzhou, China; 'bepartment of Immunology, Zhongshan School of Medicine, Sun Yat-sen University, Guangzhou, China; 'Department of Pathology, The First Affiliated Hospital of Sun Yat-sen University, Guangzhou, China; ${ }^{\mathrm{d} G u a n g d o n g}$

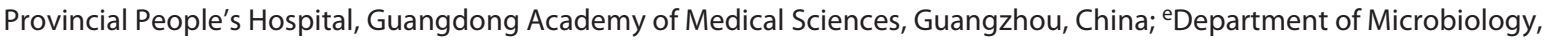
Zhongshan School of Medicine, Sun Yat-sen University, Guangzhou, China
\end{abstract}

\section{Keywords}

Long noncoding RNA · Zika virus · Interferon •

Interferon-stimulated gene $\cdot$ Innate immunity

\begin{abstract}
Expression of host noncoding RNAs and coding mRNAs is significantly altered by viral infection. In the current study, we screened the transcriptional profile of human lung epithelial A549 cells infected with Zika virus (ZIKV) by microarray assay. Seventy-nine long noncoding RNAs (IncRNAs) and 140 mRNAs were differentially expressed (DE). The bioinformatics analysis revealed that the mRNAs adjacent to the DE IncRNAs were closely related to the host responses to viral infection. We selected 7 IncRNAs from the top 50 hits for validation. The quantitative real-time PCR data confirmed that expression of selected IncRNAs was induced by ZIKV infection. Moreover, the expression of 7 IncRNAs was induced by infection of dengue virus, Japanese encephalitis virus, or vesicular stomatitis virus, or by treatment of poly $(\mathrm{I}: \mathrm{C})$ and IFN- $\beta$. Furthermore, loss of innate immune adaptor IPS-1 or receptor IFNAR1 resulted in lower induction levels of several IncRNAs by ZIKV. Overexpression of 3 IncRNAs (RPL27-OT1,
\end{abstract}

karger@karger.com www.karger.com/jin

Karger!

BOPEN ACCESS
(C) 2021 The Author(s)

Published by S. Karger AG, Basel

This is an Open Access article licensed under the Creative Commons Attribution-NonCommercial-4.0 International License (CC BY-NC) (http://www.karger.com/Services/OpenAccessLicense), applicable to the online version of the article only. Usage and distribution for commercial purposes requires written permission.
OASL-IT1, and REC8-OT3) reduced the virus yields of ZIKV. Knockout of OASL-IT1 significantly enhanced ZIKV replication. In OASL-IT1 knockout cells, the levels of interferons (IFNs) and the activation of 3 innate immune signaling pathways triggered by ZIKV were dramatically reduced. Collectively, our work found a positive feedback loop in the IFN system, in which IFNs and OASL-IT1 regulate each other, thereby promoting establishment of antiviral defense.

(c) 2021 The Author(s)

Published by S. Karger AG, Basel

\section{Introduction}

Zika virus (ZIKV) is an emerging arbovirus belonging to the Flavivirus genus, which is a large family of enveloped, single positive-stranded RNA viruses including dengue virus (DENV), Japanese encephalitis virus (JEV), yellow fever virus, and West Nile virus $[1,2]$. ZIKV is mainly transmitted to humans through the skin by the bite of infected mosquitoes [1]. Unlike other flaviviruses, ZIKV can be also transmitted by blood-borne, maternalfetal, sexual, and potentially through urine or saliva trans- 
mission [3]. The infection of ZIKV often leads to mild and self-limiting illness with fever, rash, joint pain, and conjunctivitis [4], but it is also associated with severe neurologic diseases, including microcephaly in neonates born from infected mothers and Guillain-Barré syndrome in adults [5].

The fate of ZIKV infection in an individual is largely determined by the host-virus interaction [6]. Particularly, the innate immune response mediated by interferons (IFNs) controls viral spread and disease development [7]. Upon ZIKV infection, membrane-bound Toll-like receptors or cytosolic RIG-I-like receptors recognize the viral pattern-associated molecular patterns such as its genome RNAs and activate the signal [8]. Then, the signal is transmitted through adaptor proteins, such as TIR-domaincontaining adapter-inducing interferon-beta or interferon promoter stimulator 1 (IPS-1), followed by activation of transcription factors including MAPK, NF- $\kappa \mathrm{B}$, and IRF3/7. Ultimately, the production of IFNs and proinflammatory cytokines is rapidly induced. The IFN system establishes a defense through stimulating the expression of antiviral interferon-stimulated genes (ISGs) $[9,10]$. In the murine infection model, the mice lacking the interferon $\alpha / \beta$ receptor (Ifnar $1^{-/}$) are more susceptible to ZIKV infection and develop neurological disease and succumb to ZIKV infection $[11,12]$.

So far, $>300$ protein-coding genes have been identified to be ISGs, such as IFN-inducible transmembrane (IFITM) family [13, 14], IFI6 [15], viperin [16, 17], C19orf66 [18], and $2^{\prime}, 5^{\prime}$-oligoadenylate synthetase 2 [19]. Apart from protein-coding genes, noncoding RNAs (ncRNAs) are another group of genes stimulated by IFNs [20-23]. Based on their length, ncRNAs are divided into short ncRNAs (such as miRNAs and piRNAs) and long ncRNAs (lncRNAs) that are $>200$ nucleotides in length. LncRNAs are capped and polyadenylated; lncRNAs are highly species- and tissuespecific [24]. LncRNAs are involved in a variety of cellular processes such as cell cycle, differentiation, apoptosis, development, and immune response, by interacting with RNA, DNA, or protein, and hence regulating the transcription or translation of target genes $[25,26]$.

LncRNAs are highly inducible under physiological conditions including viral infection [22, 27, 28]. Approximately $500 \mathrm{lncRNAs}$ were differentially expressed (DE) in response to infection of severe acute respiratory syndrome coronavirus and enterovirus 71 [29]. In turn, DE lncRNAs modulate the interaction between host and virus [30], such as lncRNA-ACOD1 [31], GAS5 [32], NRAV [33], lnc-ISG20 [34], and PSMB8-AS1 [35]. Recently, 2 groups investigated the expression profile of host
lncRNAs in human neural progenitor cells (hNPCs) [36] and Aedes aegypti mosquitoes upon ZIKV infection [37]. In the ZIKV-infected hNPCs, 149 DE lncRNAs were identified [36]; and in mosquitoes, expression of $486 \mathrm{ln}$ cRNAs was altered by ZIKV infection [37]. Although some lncRNAs were predicted to potentially modulate cell cycle, apoptosis, and immune response, the biological significance of DE lncRNAs have not been illustrated.

In this study, we performed a microarray assay to screen the host lncRNA expression profile in a ZIKV-permissive cell line (human lung carcinoma epithelial A549). A549 cells are widely used in ZIKV field and amount high level of innate immune response against the Flavivirus infection $[38,39]$. We found that expression of $79 \mathrm{ln}$ cRNAs and 140 mRNAs was altered by ZIKV infection. Expression of selected lncRNAs and mRNAs in different cells or in response to different viruses, dsRNA, or IFNs was further analyzed by qRT-PCR. We found that induction of several lncRNAs depends on innate immune response. Among these lncRNAs, OASL-IT1 exhibits a significant antiviral activity and positively modulates the innate immune response. These data indicated that OASL-IT1 is a new ISG, which in turn acts to promote the IFN production.

\section{Materials and Methods}

\section{Cell Culture}

Human lung carcinoma epithelial cells (A549, ATCC CCL185), human glioblastoma cells (LN229, ATCC CRL-2611), and African green monkey kidney cells (Vero, ATCC CCL-81) were maintained in Dulbecco's Modified Eagle's Medium (DMEM) supplemented with 5 or $10 \%$ fetal bovine serum (FBS) (Gibco) at 37 . $\mathrm{C}$ with $5 \% \mathrm{CO}_{2}$. The media were added with 100 units $/ \mathrm{mL}$ of streptomycin and penicillin (Invitrogen). Peripheral blood mononuclear cells (PBMCs) of healthy donors were isolated by Ficoll density gradient centrifugation. The monocytes were purified from PBMCs by anti-human CD14 magnetic particles (BD Biosciences). $\mathrm{CD}_{1} 4^{+}$monocytes were differentiated into macrophages by incubation in the RPMI-1640 medium (Invitrogen) with $10 \%$ FBS (Gibco) and 50- ng/mL human GM-CSF (Proteintech), and media were replaced every 3-4 days. After incubation for 7-10 days, monocyte-differentiated macrophages (MDMs) were obtained and used for subsequent experiments as described previously [40].

Viruses, Viral Infection, and Viral Titration

The ZIKV (H/PF/2013 strain), JEV (14-14-2 vaccine strain), and DENV2 New Guinea C were provided by Guangzhou Centers for Disease Control. The vesicular stomatitis virus (VSV) was kindly provided by Prof. Dongyan Jin (University of Hong Kong). Viruses were propagated in C6/36 or Vero cells. To amplify flaviviruses, C6/36 or Vero cells were infected with virus stock at MOI 0.01 . To amplify VSV, Vero cells were inoculated with virus stock 
at MOI 0.01 . When $90 \%$ of the cytopathic effect was observed, cells were frozen and thawed twice to lyse cells and release viral particles. The viral supernatants were separated by centrifugation. Virus stocks were titered on Vero cells by standard plaque assay as described previously [41-43] and stored at $-80^{\circ} \mathrm{C}$.

Cells were infected with ZIKV, DENV2, JEV, or VSV at MOI 3 or at indicated MOIs. In the single-step virus growth assay, the supernatants were harvested at $24 \mathrm{~h}$ postinfection (p.i.) for viral titration by standard plaque assay.

\section{Microarray Analysis}

The lncRNA microarray service was performed by Shanghai Biotechnology Corporation. Total RNAs were isolated from A549 cells using TRIzol reagent (Life Technologies) following the manufacturer's instructions. Total RNAs were purified by using the RNeasy mini kit and RNase-Free DNase Set (Qiagen). Sample labeling and array hybridization were performed according to the Agilent One-Color Microarray-Based Exon Analysis protocol (Agilent Technology). The samples were hybridized to the Arraystar human LncRNA V6.0 microarray, including 77,103 lncRNAs and 18,853 mRNAs. Three independent experiments were performed. Data were extracted with Feature Extraction software 10.7 (Agilent Technologies). Raw data were normalized by the quantile algorithm and limma packages in R. After quantile normalization, the data processing was performed with the GeneSpring GX version 12.1 software package (Agilent Technologies). Genes whose expression was changed by at least 2 -fold $(p<0.05)$ were called as differentially expressed. The lncRNAs and mRNAs were annotated through the Affymetrix Power Tools from the database of GENCODE v21/Ensembl (18,100), LNCipedia v3.1 (49,328), LncRNAdb (32), NONCODE v4 (10,079), and UCSC $(25,919)$. The microarray data were deposited in NCBI Gene Expression Omnibus (GEO) database, and the GEO accession number is GSE124094 (GPL25961).

Functional Annotation of Differentially Expressed Genes

DAVID Bioinformatics Resources (version 6.7, http://david. abcc.ncifcrf.gov/) was used to perform functional annotation enrichment and to assess the enrichment of input genes with gene ontology (GO) terms and Kyoto Encyclopedia of Genes and Genomes (KEGG) pathway analysis.

\section{Quantitative Real-Time PCR Assay}

Total RNAs were extracted using TRIzol reagent (Invitrogen), followed by DNase I digestion according to the manufacturer's protocol. cDNA was synthesized from total RNAs using HiScript II Reverse Transcriptase (Vazyme). The qRT-PCR was performed with a LightCycler 480 SYBR Green I Master in a Bio-Rad CFX96 machine. qRT-PCR primer sequences are listed in online suppl. Table 2 (see www.karger.com/doi/10.1159/000513606 for all online suppl. material). Data analyses for differences in gene expression by qRT-PCR were performed by using $\triangle \mathrm{CT}$ values as described previously [44].

\section{dsRNA or IFN Treatment}

A549 cells were transfected with $0.4 \mu \mathrm{g} / \mathrm{mL}$ poly(I:C) (Sigma) by Lipofectamine 2000 (Invitrogen) for $12 \mathrm{~h}$ or treated with 100 units/mL IFN- $\beta$ (Proteintech) for $20 \mathrm{~h}$.

\section{Plasmid Construction and Transfection}

The sgRNAs targeting OASL-IT1 were inserted into the lentiCRISPR v2 vector (Addgene, \#52961). All primer and sgRNA sequences were listed in online suppl. Table 3. LncRNAs were amplified by $5^{\prime}$ - and $3^{\prime}$-RACE PCR using the SMARTer RACE cDNA amplification kit (Takara). The PCR fragments were cloned into the pcDNA3.1(+) vector. To test the encoding ability of OASL-IT1, OASL-IT1 fused to HA tag was amplified by PCR and inserted into pcDNA3.1.

The shRNAs targeting OASL-IT1 were cloned into pLKO.1TRC (Addgene, \#10878) vector. The sequences of shRNAs were listed in online suppl. Table 3 . The sequences of plasmids were verified by sequencing.

\section{Generation of IncRNA-Expressing Cells}

The pcDNA3.1(+)-lncRNA plasmids were transfected into A549 cells by Lipofectamine 2000 (Invitrogen). The lncRNA-expressing cells were selected by G418 for 7-10 days.

Generation of OASL-IT1 Knockout and Knockdown Cells

293T cells were transfected with lentiCRISPR carrying OASLIT1 sgRNA or PLKO.1-OASL-IT1 together with pSPAX2 and pMD2.G using FuGENE ${ }^{\circledR}$ HD Transfection Reagent (Promega). Supernatants were collected at 2 days posttransfection and applied to transduce A549 cells. Knockout or knockdown cells were selected by puromycin and limiting dilution cloning. To validate the knockout effect, genomic DNA of cloned cells was extracted using a cell genomic DNA extraction kit (Bioteke). Regions surrounding sgRNA target sequences were amplified by PCR and cloned into pMD-18T (Takara) for sequencing. The efficiency of gene knockdown in cells was measured by qRT-PCR.

\section{Western Blot}

Whole cell extracts were prepared in RIPA lysis buffer. Proteins were separated on SDS-PAGE and transferred onto nitrocellulose membranes. Membranes were blocked in PBST with 5\% BSA, followed by incubation with anti-ZIKV envelope (BioFront) and anti-GAPDH (Proteintech), and then with secondary antibodies including IRDye 800 CW-conjugated anti-rabbit IgG and IRDye $680 \mathrm{CW}$-conjugated antimouse IgG secondary antibody (LI-COR). Immunoreactive bands were visualized using an infrared imaging system (Odyssey).

\section{Isolation of Cytoplasmic and Nucleic RNAs}

Cells were harvested and resuspended in RLN buffer that lysed the cell membrane. After centrifugation at $8,000 \mathrm{rpm}$ at $4^{\circ} \mathrm{C}$, supernatants and pellets were collected for cytoplasmic and nucleic RNA extraction, respectively. Cytoplasmic RNA extraction was performed using RNeasy Mini Kit (Qiagen). Nucleic RNA extraction was conducted using TRIzol reagent (Invitrogen). qRT-PCR was performed to measure the levels of $\beta$-actin, U6, and OASL-IT1 (primer sequences are listed in online suppl. Table 3 ).

\section{Immunofluorescence Microscopy}

Cells were infected with mock or ZIKV at an MOI of 3. At $24 \mathrm{~h}$ p.i., cells were washed with PBS and fixed in $4 \%$ ( $\mathrm{vol} / \mathrm{vol}$ ) paraformaldehyde for $30 \mathrm{~min}$. Cells were permeabilized in $0.02 \%$ Triton X-100 and blocked in blocking buffer for $1 \mathrm{~h}$, followed by incubation with IRF3 antibody at $4^{\circ} \mathrm{C}$ overnight. Cells were incubated with Cy3-conjugated goat antirabbit IgG (Millipore) for $1 \mathrm{~h}$. Cells were then stained with Hoechst (Invitrogen) and visualized using a Leica fluorescence microscope. 
Table 1. Information of 7 selected differentially expressed lncRNAs

\begin{tabular}{llllll}
\hline Id & Database & Classification & Style & Type & \multirow{2}{*}{ Name } \\
\hline lnc-KAT2A-1:1 & LNCipedia & Exonic sense & $\mathrm{Up}$ & Noncoding & KAT2A-OT1 \\
lnc-RPL27-2:1 & LNCipedia & Exonic sense & $\mathrm{Up}$ & Noncoding & RPL27-OT2 \\
ENST00000610643 & Ensembl & Intronic & $\mathrm{Up}$ & Noncoding & OASL-IT1 \\
lnc-RP11-468E2.4.1-3:2 & LNCipedia & Exonic sense & $\mathrm{Up}$ & Noncoding & REC8-OT3 \\
lnc-FAM3B-8:1 & LNCipedia & Exonic sense & $\mathrm{Up}$ & Noncoding & FAM3B-OT8 \\
lnc-SP110-2:1 & LNCipedia & Exonic sense & $\mathrm{Up}$ & Noncoding & SP110-OT2 \\
lnc-CH25H-2:4 & LNCipedia & Exonic antisense & $\mathrm{Up}$ & Noncoding & CH25H-AS2
\end{tabular}

lncRNAs, long noncoding RNAs.

Statistical Analysis

All statistical analyses of viral RNA levels or viral titers were performed with an unpaired, two-tailed Student's $t$ test. Data were presented as mean \pm SD from at least 3 independent experiments. The differences were considered statistically significant when $p<$ 0.05 .

\section{Results}

\section{A Subset of IncRNAs and mRNAs Was Differentially} Expressed upon ZIKV Infection

In the pilot experiment, we found that at $24 \mathrm{~h}$ p.i. of ZIKV (MOI 8), >85\% A549 cells were positively infected (online suppl. Fig. 1). Then, we harvested the mock- and ZIKV-infected cell samples and screened the expression profile of lncRNAs and mRNAs by microarray assay. Scatter plot showed the variation of lncRNA and mRNA expression between mock- and ZIKV-infected A549 cells (Fig. 1a). The heatmap showed that $79 \mathrm{lncRNAs}$ and 140 mRNAs were DE between 2 groups (criteria: fold change $>2$; $p<0.05$ ) (Fig. 1b). Seventy-two lncRNAs were upregulated, whereas 7 lncRNAs were downregulated. Seventy-nine DE IncRNAs were located on most of the chromosomes, except the chromosomes 9, 13, and 20 (Fig. 1c). They belonged to 5 categories of lncRNAs: intergenic (25.32\%), bidirectional (2.35\%), exonic sense (54.43\%), exonic antisense (13.92\%), and intronic (3.8\%) lncRNAs (Fig. 1d).

To predict potential functions of DE IncRNAs, we carried out a functional annotation of the mRNAs adjacent to these lncRNAs $[45,46]$. GO analysis showed that these mRNAs were mainly involved in the interaction between host and virus, including the response to virus, type I interferon signaling pathway, negative regulation of viral genome replication, interferon-gamma-mediated signaling pathway, innate immune response, and positive regu- lation of RIG-I signaling pathway (Fig. 1e). The KEGG pathway analysis revealed that these mRNAs were enriched in the pathways associated with infection of viruses including hepatitis $\mathrm{C}$ virus, herpes simplex virus, and influenza A virus (Fig. 1f). Overall, these data suggested that the mRNAs adjacent to the DE lncRNAs were closely related to the host response to viral infection.

\section{Validation of Differential Expression of $m R N A$ s and}

IncRNAs Induced by ZIKV

From the microarray list, we selected 7 IFN-stimulated genes, including DHX58, IFI35, MX1, OAS1, OASL, PKR, and $S P 110$, serving as positive controls for the microarray screen. Seven lncRNAs (KAT2A-OT1, PL27-OT1, OASLIT1, REC8-OT3, FAM3B-OT8, SP110-OT1, and CH25HAS2) (Table 1) were selected based on the following criteria: (1) among the top $50 \mathrm{DE}$ lncRNAs (online suppl. Table 1); (2) not annotated before; (3) detectable by qRTPCR. As expected, induction of all ISG genes by ZIKV was observed, consistent with the microarray data (Fig. 2a). Expression levels of all lncRNAs were evidently upregulated in the ZIKV-infected cells, ranging from 3.0 to 2,330.1 folds compared to the mock-infected cells (Fig. 2b). In the kinetic assay, the expression of selected lncRNAs kept increasing with the infection time extended, peaking at $36 \mathrm{~h}$ p.i. (online suppl. Fig. 2a). Their levels were also correlated with the doses of ZIKV (online suppl. Fig. 2b), indicating that the expression of selected lncRNAs was triggered by ZIKV in a time- and dose-dependent manner.

As the expression of lncRNAs is generally cell-specific, we tested whether expression of selected lncRNAs was induced in another 2 ZIKV-permissive cell lines, human glioblastoma cells LN229 cells and MDMs. As shown in Figure 2c, the expression of most lncRNAs with the exception of RPL27-OT1 in LN229 cells was significantly 


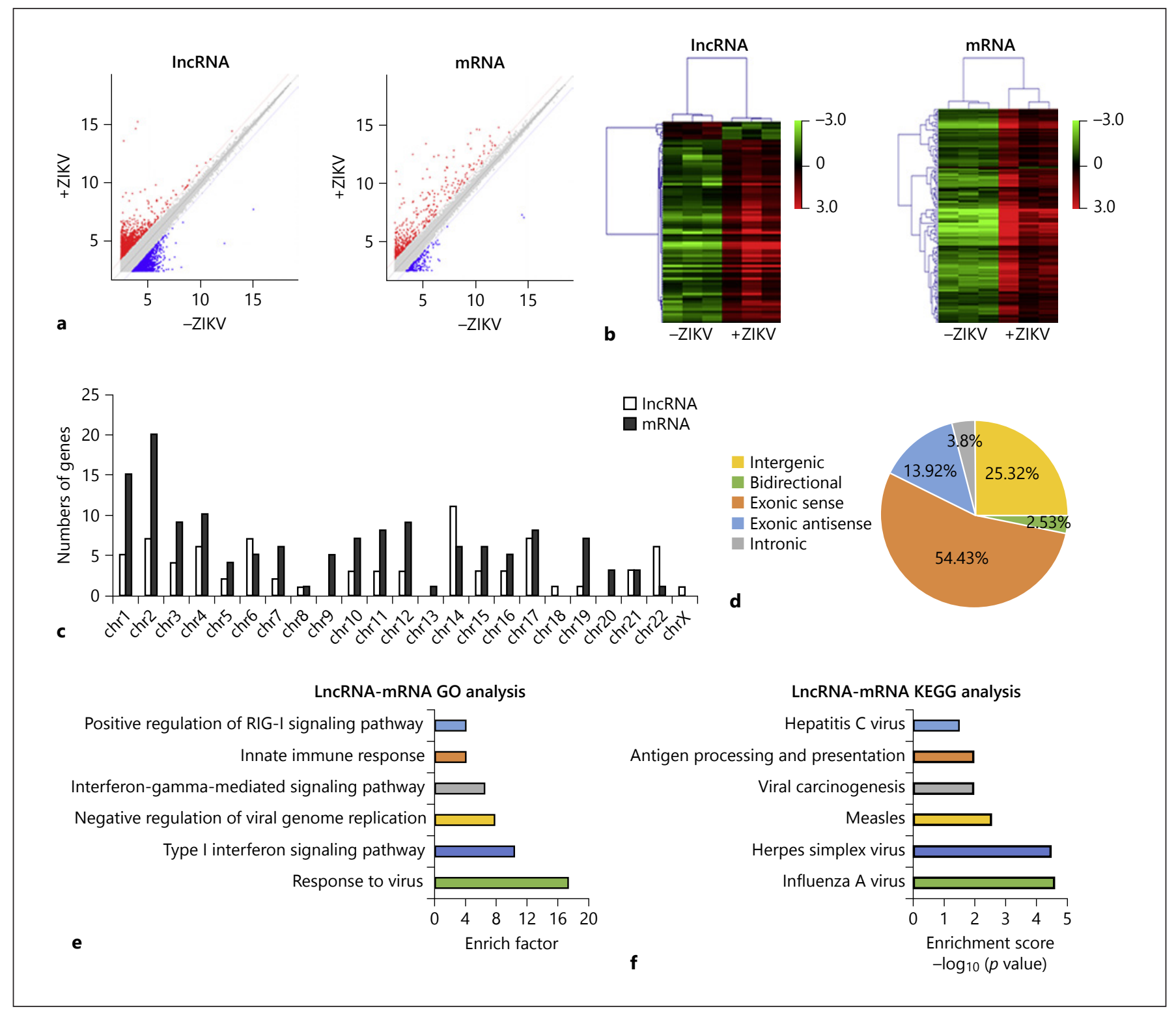

Fig. 1. Bioinformatics analysis of lncRNAs and mRNAs. a Scatter plot to show variation in lncRNA and mRNA expression. The values of $x$ and $y$ axes were the mean normalized signal values in each group ( $\log _{2}$-scaled). The red plots indicate the upregulated genes, and the blue plots indicate the downregulated genes in scatter plots. b Heat maps to show differentially expressed lncRNAs and mRNAs between mock- and ZIKV-infected groups with fold change $\geq 2.0$ and $p$ value $\leq 0.05$. The red color indicates relatively

enhanced by ZIKV infection (Fig. 2c). In human MDMs, ZIKV infection also led to notable induction of most lncRNAs except REC8-OT3 (Fig. 2c). These data suggested that the induction of selected lncRNAs by ZIKV was not restricted to a cell type. higher expression, and the green color indicates relatively lower expression. c Chromosome distribution of differentially expressed lncRNAs and mRNAs. d Classification of differentially expressed lncRNAs. GO (e, f) and KEGG pathway enrichment analysis (e) of protein-coding genes close to the differently expressed lncRNAs (f). IncRNAs, long noncoding RNAs; ZIKV, Zika virus; GO, Gene Ontology; KEGG, Kyoto Encyclopedia of Genes and Genomes.

Next, we examined whether these lncRNAs were responsive to other viruses using another 2 flaviviruses (DENV2 and JEV) and a negative single-stranded RNA virus (VSV). A549 cells were infected with mock or virus for $12 \mathrm{~h}$ p.i. (VSV) or $24 \mathrm{~h}$ p.i. (DENV2 and JEV). Total 


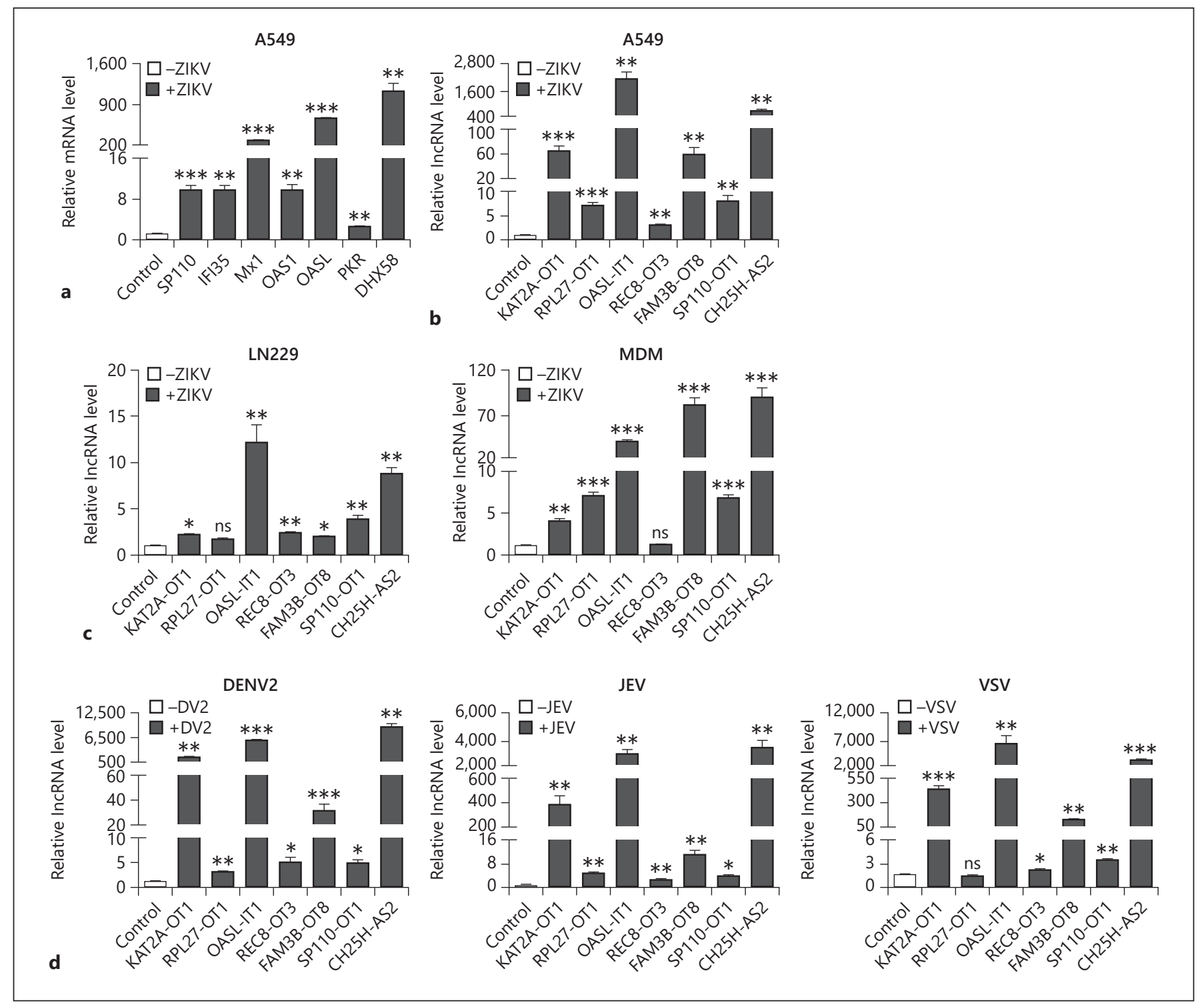

Fig. 2. Expression levels of mRNAs and lncRNAs. a, b A549 cells were infected with ZIKV for $24 \mathrm{~h}$ at MOI 8. The expression levels of 7 mRNAs (a) and 7 lncRNAs (b) were measured by qRT-PCR. c LN229 and MDMs were infected with ZIKV at MOI 8. d A549 cells were infected with DENV2, JEV, or VSV at MOI 8. Mock- and virus-infected cells were collected at $24 \mathrm{~h}$ p.i. except VSV-infected cells at $12 \mathrm{~h}$ p.i. Total RNAs were extracted for qRT-PCR to detect

RNAs were extracted for qRT-PCR. The data showed that the expression of all lncRNAs was significantly induced by DENV2 and JEV (Fig. 2d). In the VSV-infected cells, the expression of most lncRNAs, except RPL27-OT1 and REC8-OT3, was enhanced by 2.6-4,686.6 folds (Fig. 2d). These observations indicated that selected lncRNAs are responsive to other viral infection. the levels of 7 lncRNAs. U6 level was set as an internal control. The data were presented as mean $\pm \mathrm{SD}$ of at least 3 independent experiments. ${ }^{*} p<0.05 ;{ }^{* *} p<0.01 ;{ }^{* * *} p<0.001$, unpaired, two-tailed Student's $t$ test. ns, no statistical significance; lncRNAs, long noncoding RNAs; ZIKV, Zika virus; MDMs, monocyte-differentiated macrophages; DENV2, dengue virus; JEV, Japanese encephalitis virus; VSV, vesicular stomatitis virus; p.i., postinfection.

\section{Expression of Selected lncRNAs Was Induced by Poly(I:C) and IFN- $\beta$}

As our data above showed that both positive and negative single-stranded RNA viruses induce the expression of selected lncRNAs, we further explored whether they are regulated by dsRNA [47]. A549 cells were transfected with a dsRNA analog, poly(I:C). At $12 \mathrm{~h}$ posttransfection, 


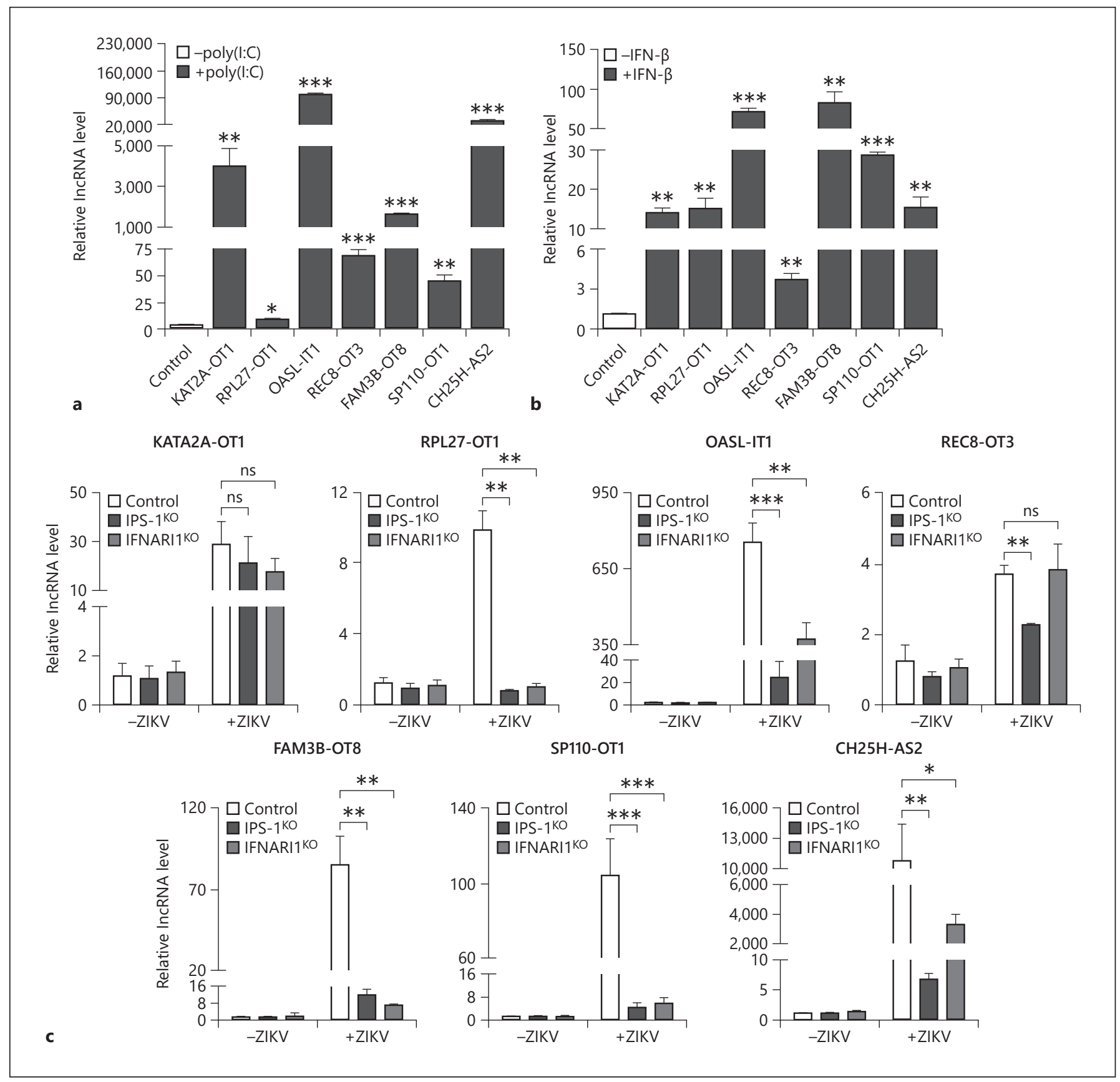

Fig. 3. Innate immune response was involved in the ZIKV-mediated induction of lncRNAs. a-c qRT-PCR to measure the levels of lncRNAs. A549 cells were transfected with poly(I:C) for $12 \mathrm{~h}$ (a) or treated with 100 units of IFN- $\beta$ for $20 \mathrm{~h}$ (b). c Control, IPS- $1^{\mathrm{KO}}$, and IFNAR $1^{\mathrm{KO}}$ cells were infected with mock or ZIKV. Cells were harvested for RNA extraction, and the expression of 7 lncRNAs was quantified by qRT-PCR. U6 level was set as an internal control.
The data were presented as mean \pm SD of at least 3 independent experiments. ${ }^{*} p<0.05 ;{ }^{* *} p<0.01 ;{ }^{* * *} p<0.001$, unpaired, twotailed Student's $t$ test. ns, no statistical significance; lncRNAs, long noncoding RNAs; ZIKV, Zika virus; IFN- $\beta$, interferon-beta; IPS- 1 , interferon promoter stimulator 1 ; IFNAR 1 , interferon $\alpha / \beta$ receptor. 


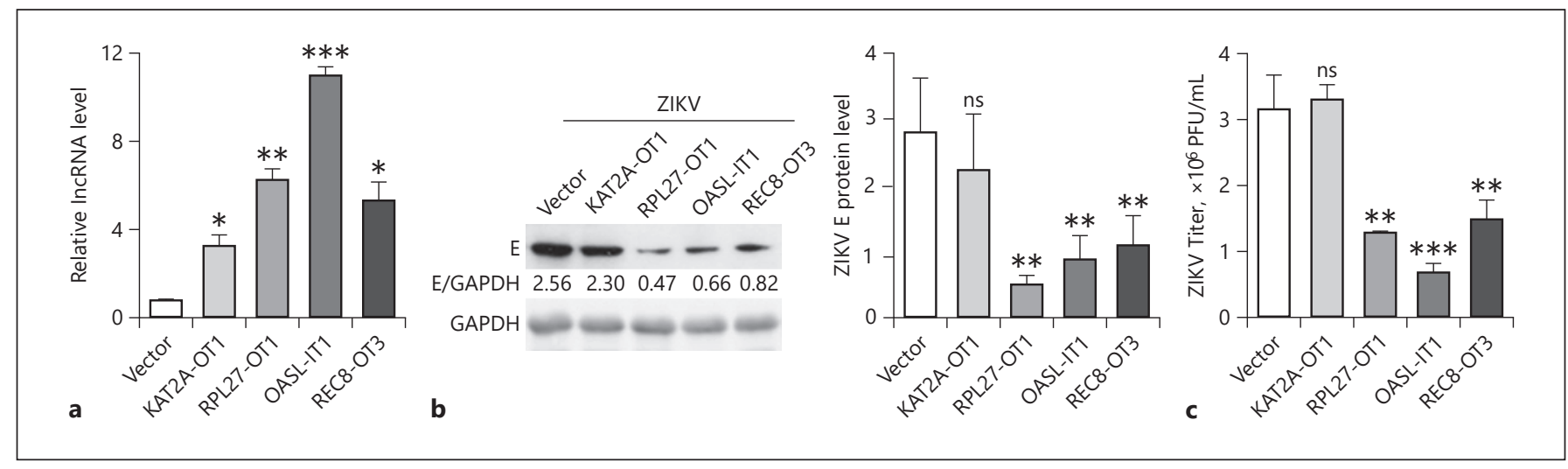

Fig. 4. Three lncRNAs restricted the ZIKV replication. a Expression levels of lncRNAs expressing A549 cells validated by qRTPCR. b, c Replication levels of ZIKV. Control and lncRNA-expressing cells were infected with ZIKV at MOI 5. Cells or supernatants were harvested at $24 \mathrm{~h}$ p.i. b The viral E protein levels were tested by Western blot. $\mathbf{c}$ The viral titers were determined by plaque assay. Data were shown as mean \pm SD of at least 3 independent experiments. ${ }^{*} p<0.05 ;{ }^{* *} p<0.01 ;{ }^{* * *} p<0.001$, unpaired, twotailed Student's $t$ test. ns, no statistical significance; lncRNAs, long noncoding RNAs; ZIKV, Zika virus; p.i., postinfection. cells were harvested for qRT-PCR. The expression of all 7 lncRNAs was upregulated by poly(I:C), ranging from approximately 5 to 70,000 folds (Fig. 3a). Considering the expression of type I IFNs was greatly induced by ZIKV in the A549 cells, we tested whether the type I IFNs are involved in the induction of lncRNAs. The qRT-PCR data showed that the IFN- $\beta$ treatment also enhanced the levels of all lncRNAs, although the induction magnitudes were generally lower than those induced by the ZIKV (Fig. 3b). This observation implies that the induction of lncRNAs might be regulated by both IFN-dependent and IFN-independent ways.

To investigate whether the induction of lncRNAs relies on the innate immune response, we employed 2 knockout cells lacking IPS-1 or IFNAR1 [7], 2 key molecules in the signaling pathway. The levels of lncRNAs in the control, IPS- $1^{\mathrm{KO}}$, and IFNAR ${ }^{\mathrm{KO}}$ cells were compared by qRT-PCR. In the mock-infected cells, the levels of all lncRNAs in control cells, IPS- $1^{\mathrm{KO}}$, and IFNAR $1^{\mathrm{KO}}$ cells were comparable (Fig. 3c). As shown in Figure 3c, the ZIKV-induced expression of most lncRNAs, except $K A T 2 A-O T 1$, in the IPS- $1^{\mathrm{KO}}$ and IFNAR $1^{\mathrm{KO}}$ cells were significantly lower than the control cells (Fig. 3c). Particularly, the induction of RPL27-OT1 in the IPS $1{ }^{\mathrm{KO}}$ and IFNAR $1^{\mathrm{KO}}$ cells was completely abolished (Fig. 3c). Taken together, we concluded that the expression of 7 lncRNAs was differentially regulated by innate immunity, among which RPL27-OT1, OASL-IT1, FAM3B-OT8, SP110-OT1, and $\mathrm{CH} 25 \mathrm{H}$-AS2 probably belong to ISGs.

\section{Three IncRNAs Restrict the Replication of ZIKV}

To explore the biologic significance of lncRNA induction, we first examined their roles in the ZIKV replication through a gain-of-function strategy. The lncRNA gene fragments were amplified and inserted into pcDNA3.1(+) vector. Among 7 lncRNAs, 4 lncRNAs (KAT2A-OT1, RPL27-OT1, OASL-IT1, and REC8-OT3) were successfully cloned into expression vector, while the full-length fragments of other 3 lncRNAs (FAM3B-OT8-7, SP110$O T 1$, and $\mathrm{CH} 25 \mathrm{H}-\mathrm{AS} 2$ ) could not be amplified, probably due to their size and secondary/tertiary structures. Following plasmid transfection, A549 bulk cells expressing lncRNA were obtained by G418 selection. The levels of individual lncRNAs in these cells were confirmed by qRT-PCR assay. They were upregulated by $4.0-13.6$ folds (Fig. 4a). Then, we compared the viral replication levels by Western blot and plaque assay. The viral E protein levels in RPL27-OT1, OASL-IT1, and REC8-OT3, but not KAT2A-OT1, expressing cells were significantly lower than that in the control cells (Fig. 4b). Consistently, the viral yields in RPL27-OT1, OASL-IT1, and REC8-OT3 expressing cells were reduced by $2.51-, 4.70-$, and 2.17 fold, respectively (Fig. 4c), suggesting that they play an antiviral role in ZIKV infection.

\section{OASL-IT1 Does Not Encode a Protein}

In the following assays, we focused on OASL-IT1 as it confers the strongest antiviral activity. Human OASL-IT1 is located on the reverse strand of chromosome 12 from positions 121019111 to 121019485 (375 bp) and is in the 


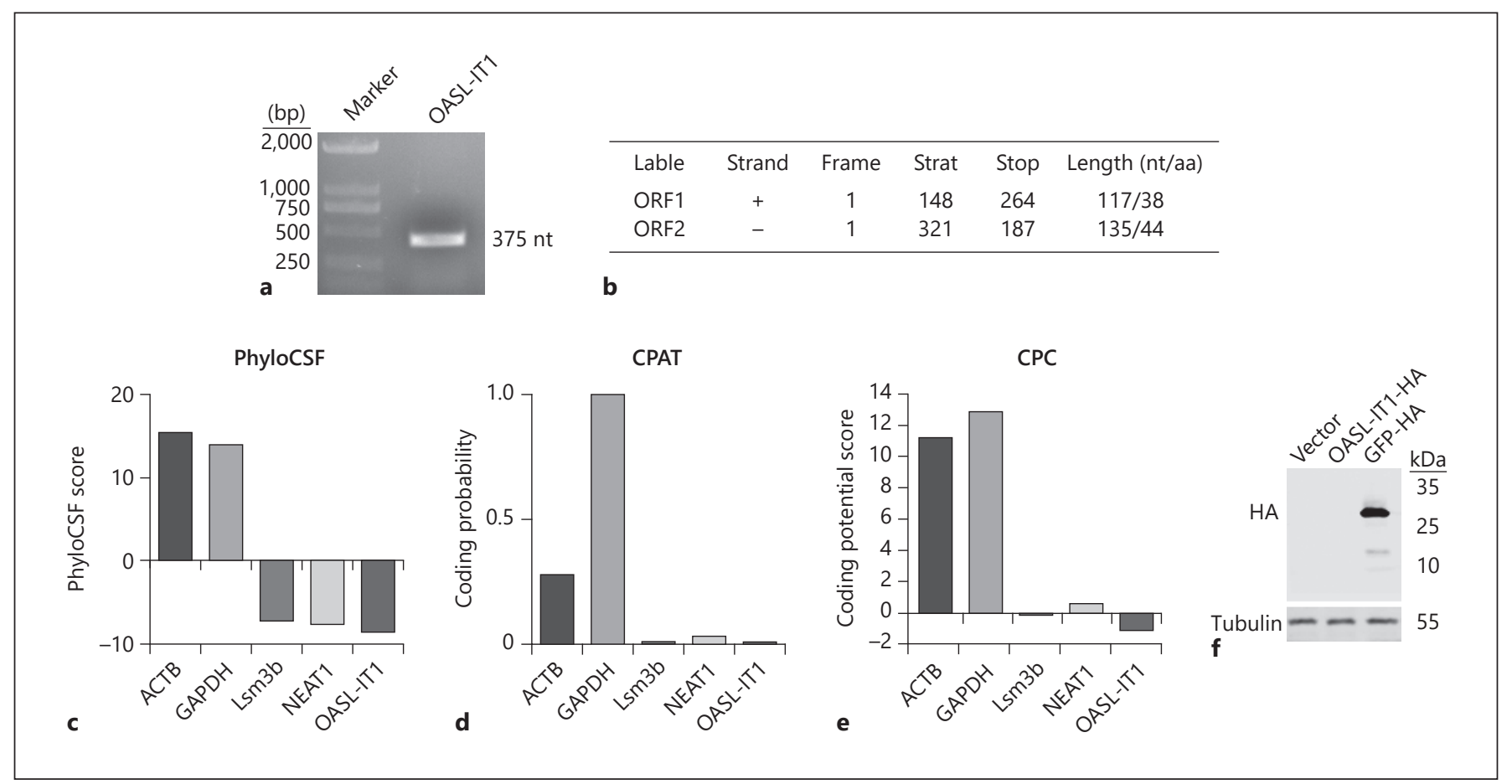

Fig. 5. Characterization of OASL-IT1. a RACE assay to determine the size of full-length $O A S L-I T 1$. b-e Prediction of coding ability of OASL-IT1. ORF Finder (b), PhyloCSF (c), CPAT (d), and CPC (e) were employed to predict the coding probability of OASL-IT1. $A C T B$ and GAPDH served as control coding genes, and $L s m 3 b$ and NEAT1 served as control noncoding genes. f Western blot. 293 cells were transfected with pcDNA3.1 vector, pcDNA3.1-OASLIT1-HA, or pcDNA3.1-GFP-HA plasmid. At $24 \mathrm{~h}$ posttransfection, cells were harvested for Western blot using anti-HA antibody. Blots were representative of at 3 independent experiments. CPAT, Coding Potential Assessment Tool; CPC, Coding Potential Calculator. intron 5 of OASL. Human OASL-IT1 shares 93.6\% homology with rhesus, but no homologous gene was identified in mouse, dog, and elephant, suggesting that it is highly species-specific (online suppl. Fig. 3). The size of full-length $O A S L-I T 1$ was measured by RACE. The DNA gel showed a specific band in the length of $375 \mathrm{bp}$, consistent with the microarray data (Fig. 5a). To explore the coding possibility of OASL-IT1, we utilized online prediction tools, including ORF Finder, PhyloCSF, Coding Potential Assessment Tool (CPAT), and Coding Potential Calculator (CPC). The ORF Finder predicted that 2 potential ORFs of OASL-IT1 are shorter than $150 \mathrm{nt}$, thus unlikely to encode a protein (Fig. 5b). The PhyloCSF analysis indicated that the OASL-IT1 yielded negative score to encode protein as other lncRNAs, $L s m 3 b$ and NEAT1, while 2 protein-coding genes, $A C T B$ and GAPDH, yielded positive scores (Fig. 5c). Similarly, the CPAT and CPC analyses predicted that OASL-IT1 possesses an extremely low coding potential compared to $A C T B$ and GAPDH (Fig. 5d, e). To experimentally vali- date these predictions, we constructed a eukaryotic expression vector expressing full length of OASL-IT1 fused to HA tag (pcDNA3.1-OASL-IT1-HA). The Western blot data showed no band was detectable in the cells transfected with pcDNA3.1-OASL-IT1-HA. In contrast, a specific band was seen in the pcDNA3.1-GFP-HA-transfected cells (Fig. 5f). These data demonstrated that OASL-IT1 does not possess protein-coding ability.

\section{Depletion of OASL-IT1 Leads to an Increased Viral Replication}

Next, we performed a fraction assay to examine the subcellular localization of OASL-IT1. OASL-IT1 was present in both nuclei and cytoplasm, and upon viral infection, the levels of both nucleic and cytoplasmic OASLIT1 were gradually increased (Fig. 6a). As a control, U6 and actin were mainly present in the nuclei or cytoplasm, respectively (Fig. 6a).

To further confirm the role of OASL-IT1 in the ZIKV replication, we employed 2 loss-of-function strategies in- 


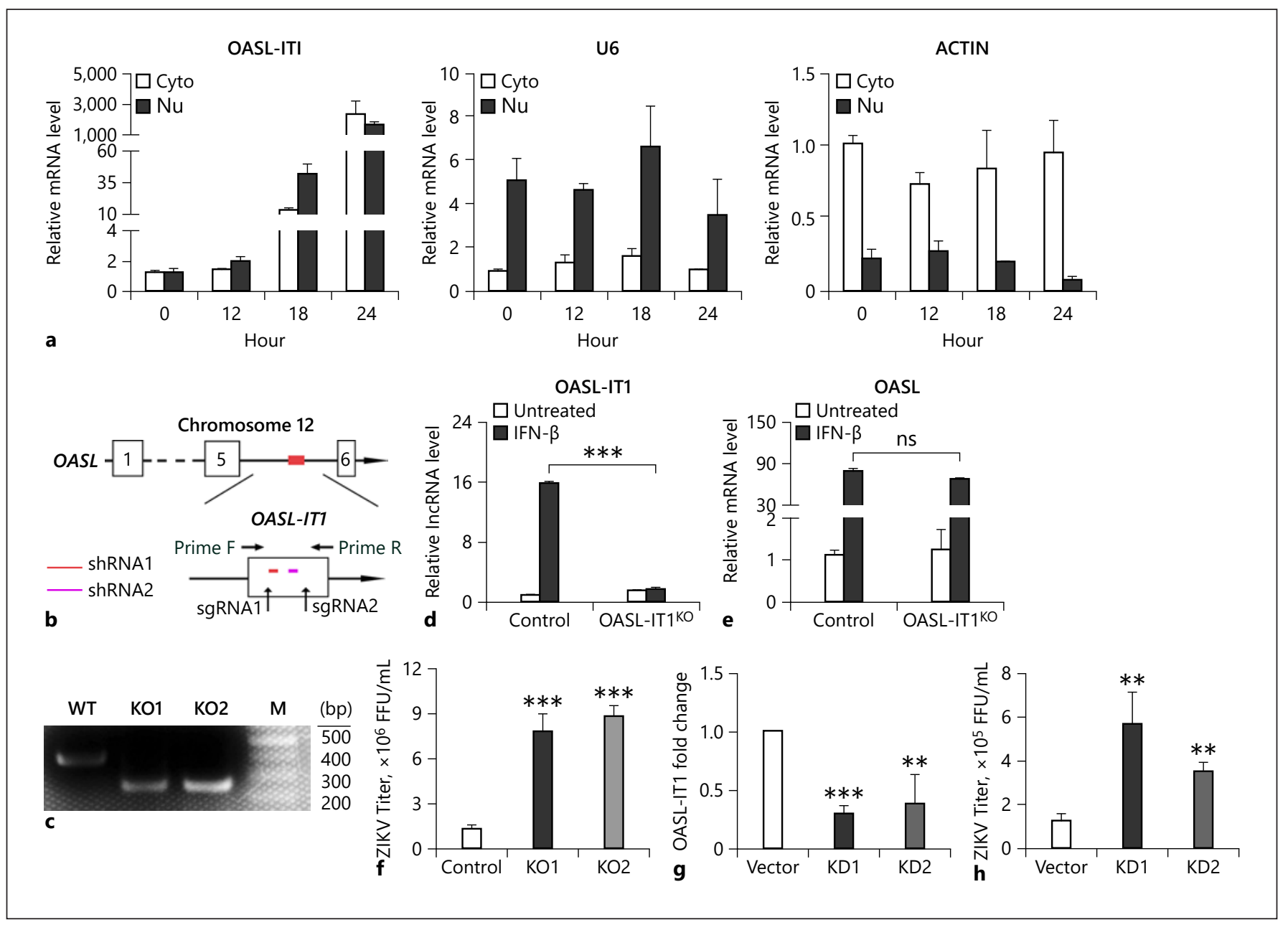

Fig. 6. OASL-IT1 played an antiviral role. a Subcellular distribution of OASL-IT1. A549 cells were infected with ZIKV at MOI 5 and harvested at $0,6,12$, and $24 \mathrm{~h}$ p.i. for subcellular fractionation and RNA extraction. qRT-PCR was performed to measure the levels of OASL-IT1, U6, and actin, respectively. b Schematic diagram of CRISPR/Cas9 knockout strategy. The positions of OASL-IT1, sgRNA1, sgRNA2, and PCR primers used to amplify the OASL-IT1 fragment were indicated. c DNA gel to show the PCR fragments amplified from the genomic DNA of WT and 2 OASL-IT ${ }^{\mathrm{KO}}$ cells. d, e mRNA levels of OASL-IT1 and OASL. Control and OASLIT $1^{\mathrm{KO}}$ cells were treated with IFN- $\beta$ for 20 h. Total RNA was extracted for qRT-PCR to measure the levels of OASL-IT1 and OASL. f Plaque assay. Control and OASL-IT1 ${ }^{\mathrm{KO}}$ cells were infected with ZIKV at MOI 5. At $24 \mathrm{~h}$ p.i., supernatants were harvested for plaque assay. $\mathbf{g}, \mathbf{h}$ Impact of OASL-IT1 knockdown on the viral replication. Control and OASL-IT1 ${ }^{\mathrm{KD}}$ cells were infected with ZIKV at MOI 5. Total RNAs were prepared for qRT-PCR $(\mathbf{g})$ to measure the levels of OASL-IT1. U6 was set as an internal control. h Supernatants were harvested at $24 \mathrm{~h}$ p.i. for plaque assay. Data were shown as mean \pm SD of at least 3 independent experiments. ${ }^{*} p<0.05 ;{ }^{* *} p<0.01 ;{ }^{* * *} p<0.001$, unpaired, two-tailed Student's $t$ test. ns, no statistical significance; ZIKV, Zika virus; p.i., post infection; WT, wild type. cluding CRISPR/Cas9 gene editing-based knockout and RNAi-based knockdown. Lentivirus carrying 2 different sgRNAs targeting OASL-IT1 (Fig. 6b) were transduced into A549 cells and selected by puromycin. Genomic DNAs of individual cell clones were prepared for PCR and DNA sequencing. The DNA gel showed that a 143-nt fragment was removed out of OASL-IT1 gene in 2 cell clones, which were designated as OASL-IT1 ${ }^{\mathrm{KO}}$ (Fig. 6c). To test whether the editing of OASL intron 5 affects the $O A S L$ expression, we compared the basal and IFN-inducible levels of OASL in the control and OASL-IT1 ${ }^{\mathrm{KO}}$ cells. As expected, the level of OASL-IT1 in the OASL-IT1 $1^{\mathrm{KO}}$ cells was barely induced by IFN- $\beta$ (Fig. $6 \mathrm{~d}$ ). The OASL levels in the control and OASL-IT1 ${ }^{\mathrm{KO}}$ cells were compa- 


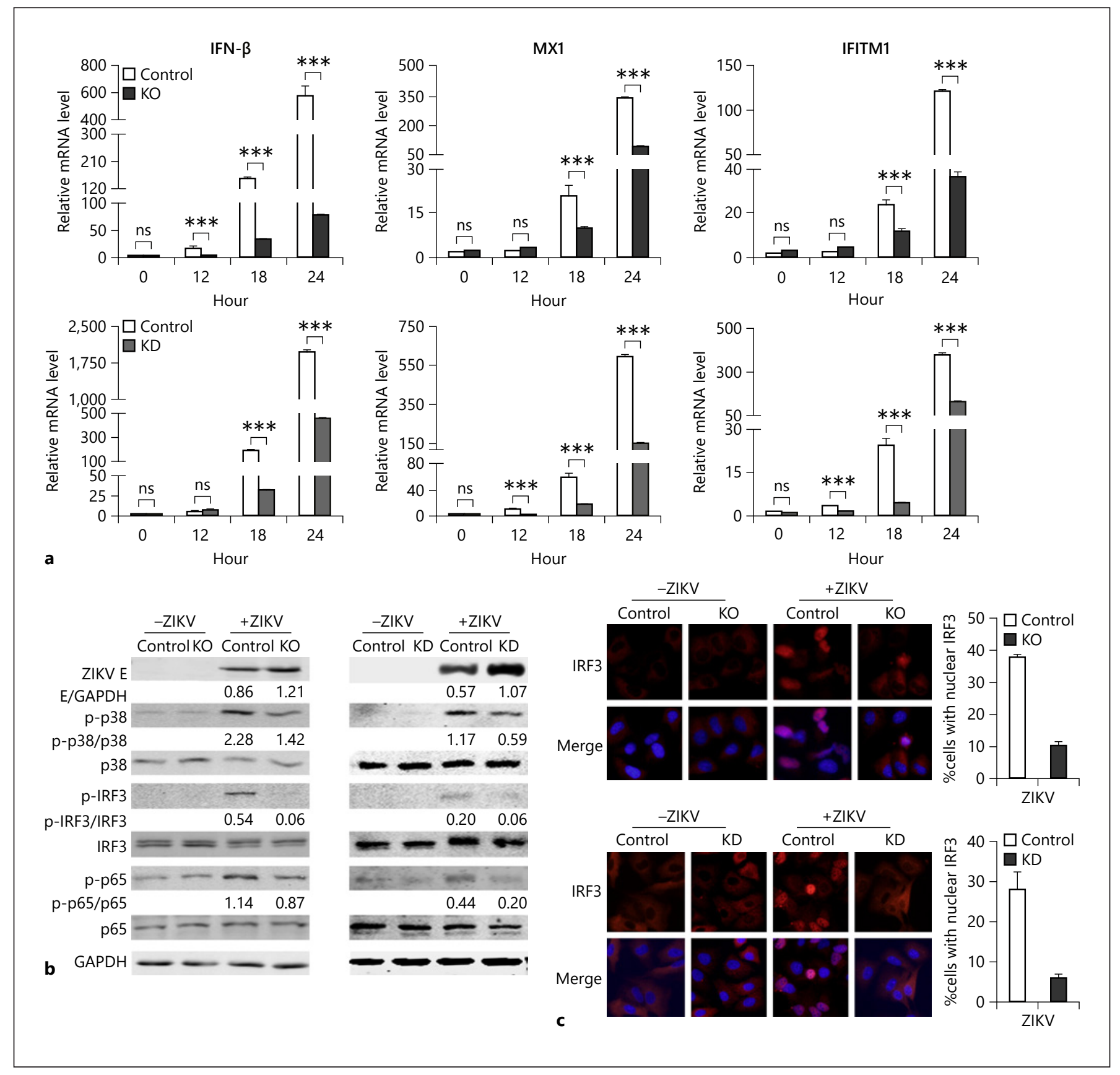

Fig. 7. OASL-IT1 promoted the innate immune response. a $\mathrm{qRT}$ PCR to detect the levels of IFN- $\beta, M X 1$, and IFITM1. The control, OASL-IT $1^{\mathrm{KO}}$, and OASL-IT1 ${ }^{\mathrm{KO}}$ cells were infected with ZIKV at MOI 5. At indicated time points, total RNAs were prepared for qRT-PCR. GAPDH level was set as an internal control. Data were shown as mean $\pm \mathrm{SD}$ of at least 3 independent experiments. *** $p<0.001$, unpaired, two-tailed Student's $t$ test. b, c Activation levels of 3 innate signaling pathways. Control, OASL-IT1 ${ }^{\mathrm{KO}}$, and OASL-IT1 ${ }^{\mathrm{KD}}$ cells were infected with ZIKV at MOI 5. At $24 \mathrm{~h}$ p.i., cells were collected for Western blot (b) or IFM (c). b Whole cell extracts were prepared and subjected to SDS-PAGE and Western blot using indicated antibodies. GAPDH was loaded as an internal control. In the IFM assay, cells were incubated with anti-IRF3 antibody (red) and DAPI (blue). Blots and cell images were representative of at 3 independent experiments. ns, no statistical significance; ZIKV, Zika virus; p.i., postinfection; IFM, immunofluorescence microscopy. 
rable in the absence and presence of IFN- $\beta$, indicating that the OASL level was not affected by the editing of OASL-IT1 (Fig. 6e).

Then, we compared the viral yields in the control and OASL-IT $1^{\mathrm{KO}}$ cells by plaque assay. The ZIKV yields in 2 OASL-IT $1^{\mathrm{KO}}$ cells were increased by $3-4$ folds $(p<0.001$, Fig. 6f). In the RNAi-based knockdown assay, A549 cells were transfected with 2 different shRNAs expressing plasmids and selected by puromycin. In 2 OASL-IT1 knockdown cell clones, the levels of OASL-IT1 were largely silenced (Fig. 6g), and the viral titers were increased by 3.6- and 2.6-fold, respectively (Fig. 6h), demonstrating that OASL-IT1 is a restriction factor of ZIKV.

\section{OASL-IT1 Promotes the Innate Immune Response}

Next, we probed whether OASL-IT1 restricts ZIKV infection by regulating the innate immune response as other ISG proteins or lncRNAs [48-51]. The kinetic levels of IFN- $\beta$ in the control, OASL-IT1 ${ }^{\mathrm{KO}}$, or OASL-IT ${ }^{\mathrm{KD}}$ cells upon ZIKV infection were measured by qRT-PCR. In the control cells, the levels of IFN- $\beta$ were progressively increased, peaking at $24 \mathrm{~h}$ p.i. (Fig. $7 \mathrm{a}$, white bars). Intriguingly, the IFN- $\beta$ levels in OASL-IT ${ }^{\mathrm{KO}}$ and OASL-IT $1^{\mathrm{KD}}$ cells were significantly lower than the control cells, ranging from 4.4 to 7.7 folds (Fig. 7a). Similarly, the levels of 2 classic ISGs (MX1 and IFITM1) in the OASL-IT1-deficient cells were significantly lower than in the OASL-IT1sufficient cells (Fig. 7a).

To dissect which signaling pathway(s) of innate immune response is modulated by OASL-IT1, we examined the activation extent of 3 major transcription factors including p38 MAPK, IRF3, and NF- $\kappa \mathrm{B}$, which are phosphorylated and translocalized into nuclei upon activation [52]. The Western blot data revealed that the total protein levels of p38, IRF3, and p65 were comparable in all samples as expected. Upon ZIKV infection, phosphorylation levels of p38, IRF3, and NF- $\kappa$ B p65 in the control cells were readily detected, but were significantly alleviated in the OASL-IT1 ${ }^{\mathrm{KO}}$ and OASL-IT1 ${ }^{\mathrm{KD}}$ cells (Fig. 7b). In the immunofluorescence microscopy assay, the IRF3 protein in the mock-infected cells was predominantly distributed in the cytoplasm (Fig. 7c). Upon ZIKV infection, around $30 \%$ of control cells showed a nuclei distribution pattern of IRF3, while $<10 \%$ of OASL-IT $1{ }^{\mathrm{KO}}$ or OASL-IT $1^{\mathrm{KD}}$ cells exhibited the nuclei translocation of IRF3. The collective data indicated that OASL-IT1 positively regulates the activation of $\mathrm{p} 38 \mathrm{MAPK}, \mathrm{IRF} 3$, and NF- $\mathrm{kB}$, eventually promoting the IFN and ISGs induction.

\section{Discussion}

Upon viral invasion, the host cell rapidly establishes an antiviral defense by eliciting the innate immune response. Although hundreds of protein-coding ISGs have been documented [9], a large proportion of noncoding RNA ISG genes remain unknown [20,21]. Current study identified and characterized several lncRNAs induced by IFNs and viruses and provided evidence that a novel lncRNA, OASL-IT1, acts as a positive regulator of IFN response and plays an antiviral role.

Our microarray data identified a subset of lncRNAs in A549 cells triggered by ZIKV, while the pattern is quite distinct from the data obtained from hNPCs [36]. The highest DE mRNAs in A549 cells are related to the response to viral infection, particularly the innate immune response including IFNs (such as IFNB1, IFNL2, and IFNL1) and ISGs (such as OASL, IFIT2, and MX1), in line with Chen's group [39]. In contrast, the upregulated gene set in hNPCs was enriched in the processes related to nucleic acid metabolism regulation [36]. Moreover, 76 out of 92 lncRNAs were downregulated in ZIKV-infected hNPCs, while only 7 out of $79 \operatorname{lncRNAs}$ were decreased in A549 cells in the present study. Considering hNPCs and A549 cells originated from different tissues, it is not surprising to find their lncRNA expression profiles are differentially regulated. To be pointed out, although the basal levels of 72 lncRNAs in A549 cells were extremely low, they are highly inducible to viral infection, suggesting these lncRNAs play some roles in the host-virus interaction.

We found that the innate immune response is crucial in the induction of many lncRNAs. Stimuli, including infection of 3 RNA viruses (DENV2, JEV, and VSV) and poly(I:C) or IFN- $\beta$ treatment, upregulate the expression of most lncRNAs. In addition, we found that the depletion of IPS-1 or IFNAR1 dramatically impaired the induction of many lncRNAs, especially RPL27-OT1, illustrating that the induction of many lncRNAs was dependent on innate immune response. Given the elevation of RPL27-OT1 occurred at later stage of viral infection (24 $h$ p.i.), we concluded that the induction of RPL27-OT1 by ZIKV is IFN-dependent. In contrast, the induction of another 6 lncRNAs in the IPS- $1^{\mathrm{KO}}$ or IFNAR $1^{\mathrm{KO}}$ cells was significantly but not completely decreased, suggesting they are modulated in both IFN-dependent and IFN-independent manner. Notably, the levels of both OASL and OASL-IT1 were enhanced by $>400$-fold ( 24 h p.i.). As OASL-IT1 is located in the intron of OASL, OASL and OASL-IT1 are probably regulated through the same 
mechanism. In future, bioinformatics and molecular analysis to map the IFN-stimulated response element in the promoter regions of these lncRNAs will advance our understanding on how IFNs regulate expression of these lncRNAs.

Functionally, 3 lncRNAs, including RPL27-OT1, OASL-IT1, or REC8-OT3, confer an antiviral activity. To be pointed out, the antiviral effect of ectopic-expressed lncRNAs was generally moderate, which might be because (1) the lncRNAs in the bulk expressing cells were not evenly expressed in each cells and (2) functions of lncRNAs are generally exerted through other molecules such as proteins, RNAs, or DNAs [20, 53]; thus, their effects on the viral replication might be delayed. LncRNA OASL-IT1 is intronic and distributed in both cytoplasm and nuclei. In the RNAi and CRISPR/Cas9 assay, the OASL-IT1 levels were successfully reduced while OASL was barely affected. In the OASL-IT1 depleted cells, we observed a significant increase in viral titer, confirming that OASL-IT1 plays a restriction role in ZIKV replication.

Intriguingly, our study revealed that the OASL-IT1 has a positive feedback on the innate immune response. In the OASL-IT1-deficient cells, the activation of 3 transcriptional factors and the levels of IFN and ISGs were greatly alleviated. As all of the signaling pathways were affected, we deduced that the OASL-IT1 most likely acts at an upstream step, such as virus recognition or IPS-1 adaptor activation, to modulate the innate response. To test this hypothesis, we have performed RNA immunoprecipitation assay to test whether OASL-IT1 binds the pattern recognition receptors (RIG-I and MDA5) and adaptor IPS-1, but no association was detected (data not shown). An unbiased screen of OASL-IT1 interacting proteins through RNA pulldown and mass spectrum assay is under investigation, which will illustrate how OASL-IT1 is involved in the innate immune response.

Taken together, we proposed that the antiviral action of OASL-IT1 is exerted, at least in part, through its positive feedback on the IFN system. The IFNs including type I IFNs and type III IFNs have been well documented to prevent ZIKV infection, through stimulating their downstream effectors such as IFITMs [7, 13, 52]. The expression of OASL-IT1 is upregulated by IFNs, suggesting that it is an IFN-stimulated gene like OASL; in turn, it augments the extent of innate immune response. Thus, OASL-IT1 and IFNs constitute a positive feedback loop. So far, this regulation loop has been found between IFNs and many ISGs [20,48-50], which is a common phenomenon of innate immune response. To be pointed out, the
OASL-IT1 gene is only conserved in primates, but not in other mammals such as mouse, implying that OASL-IT1 might have some unique functions in primates.

Overall, our work identified a number of new lncRNAs that are responsive to ZIKV infection and established a relationship between a few lncRNAs and the innate immune response. One of the new lncRNAs, OASL-IT1, in turn promotes the expression of IFNs and ISGs and restricts the replication of ZIKV. Our work further demonstrated that lncRNAs share similar characteristics with ISG proteins, underscoring the importance of lncRNAs in the host innate antiviral response.

\section{Statement of Ethics}

Experiments involving human MDMs were reviewed and approved by the local Ethics Committee of the Zhongshan School of Medicine, and were conducted ethically in accordance with the World Medical Association Declaration of Helsinki. All donors provided written informed consent. All samples were anonymized before use.

\section{Conflict of Interest Statement}

The authors declare that they have no conflicts of interest.

\section{Funding Sources}

This research was supported by the National Natural Science Foundation of China (Grant No. 31970887), Guangdong Science and Technology Department (Grant No. 2018A050506029), National Special Research Program of China for Important Infectious Diseases (2018ZX10101004003001), and Natural Science Foundation of Guangdong Province (Grant Nos. 2020A1515010570, 2020A1515010870, and 2019A1515011336).

\section{Author Contributions}

The conceptualization of this project was conducted by W.Y. and Z.P. Investigation was performed by W.Y., H.Z.T.,L.Q.S., L.Y.X., C.C.C., H.Y.X., H.C.B., and Z.J.S. Formal analysis, visualization, and original draft preparation were performed by W.Y., H.Z.T., H.J.F., L.C., and Z.P. The manuscript was reviewed and edited by L.C. and Z.P. Supervision and project administration were carried out by Z.P. and L.C., and funding acquisition was done by Z.P., L.C., H.J.F., Z.J.S., and C.C.C. All authors have read and agreed to the published version of the manuscript. 


\section{References}

1 Musso D, Gubler DJ. Zika virus. Clin Microbiol Rev. 2016 Jul;29(3):487-524.

2 Pierson TC, Diamond MS. The continued threat of emerging flaviviruses. Nat Microbiol. 2020 Jun;5(6):796-812.

3 Sakkas H, Bozidis P, Giannakopoulos X, Sofikitis N, Papadopoulou C. An update on sexual transmission of Zika virus. Pathogens. 2018 Aug 3;7(3):66

4 Pierson TC, Diamond MS. The emergence of Zika virus and its new clinical syndromes. Nature. 2018 Aug;560(7720):573-81.

5 Olagnier D, Muscolini M, Coyne CB, Diamond MS, Hiscott J. Mechanisms of Zika virus infection and neuropathogenesis. DNA Cell Biol. 2016 Aug;35(8):367-72.

6 Lee I, Bos S, Li G, Wang S, Gadea G, Despres $\mathrm{P}$, et al. Probing molecular insights into Zika virus(-)host interactions. Viruses. 2018 May 2;10(5):233

7 Xie X, Shan C, Shi PY. Restriction of Zika virus by host innate immunity. Cell Host Microbe. 2016 May 11;19(5):566-7

8 Chazal M, Beauclair G, Gracias S, Najburg V, Simon-Lorière E, Tangy F, et al. RIG-I recognizes the $5^{\prime}$ region of dengue and Zika virus genomes. Cell Rep. 2018 Jul 10;24(2):320-8.

9 Schneider WM, Chevillotte MD, Rice CM. Interferon-stimulated genes: a complex web of host defenses. Annu Rev Immunol. 2014; 32:513-45.

10 Lee AJ, Ashkar AA. The dual nature of type I and type II interferons. Front Immunol. 2018; 9:2061.

11 Lazear HM, Govero J, Smith AM, Platt DJ, Fernandez E, Miner JJ, et al. A mouse model of Zika virus pathogenesis. Cell Host Microbe. 2016 May 11;19(5):720-30.

12 Caine EA, Jagger BW, Diamond MS. Animal models of Zika virus infection during pregnancy. Viruses. 2018 Oct 31;10(11):598.

13 Savidis G, Perreira JM, Portmann JM, Meraner P, Guo Z, Green S, et al. The IFITMs inhibit Zika virus replication. Cell Rep. 2016 Jun 14;15(11):2323-30.

14 Fu B, Wang L, Li S, Dorf ME. ZMPSTE24 defends against influenza and other pathogenic viruses. J Exp Med. 2017 Apr 3;214(4):91929.

15 Richardson RB, Ohlson MB, Eitson JL, Kumar A, McDougal MB, Boys IN, et al. A CRISPR screen identifies IFI6 as an ER-resident interferon effector that blocks flavivirus replication. Nat Microbiol. 2018 Nov;3(11) 1214-23.

16 Van der Hoek KH, Eyre NS, Shue B, Khantisitthiporn O, Glab-Ampi K, Carr JM, et al. Viperin is an important host restriction factor in control of Zika virus infection. Sci Rep. 2017 Jun 30;7(1):4475.
17 Panayiotou C, Lindqvist R, Kurhade C, Vonderstein K, Pasto J, Edlund K, et al. Erratum for Panayiotou et al., "Viperin restricts Zika virus and tick-borne encephalitis virus replication by targeting NS3 for proteasomal degradation”. J Virol. 2018 Apr 1;92(12).

18 Wu Y, Yang X, Yao Z, Dong X, Zhang D, Hu $\mathrm{Y}$, et al. C19orf66 interrupts Zika virus replication by inducing lysosomal degradation of viral NS3. PLoS Negl Trop Dis. 2020 Mar; 14(3): $\mathrm{e} 0008083$.

19 Liao X, Xie H, Li S, Ye H, Li S, Ren K, et al. $2^{\prime}, 5^{\prime}$-Oligoadenylate synthetase 2 (OAS2) inhibits Zika virus replication through activation of type Iota IFN signaling pathway. Viruses. 2020 Apr 8;12(4):418.

20 Carpenter S. Long noncoding RNA: novel links between gene expression and innate immunity. Virus Res. 2016 Jan 2;212:137-45.

21 Ouyang J, Hu J, Chen JL. IncRNAs regulate the innate immune response to viral infection. Wiley Interdiscip Rev RNA. 2016 JanFeb;7(1):129-43.

22 Valadkhan S, Gunawardane LS. IncRNA-mediated regulation of the interferon response. Virus Res. 2016 Jan 2;212:127-36.

23 Valadkhan S, Plasek LM. Long non-coding RNA-mediated regulation of the interferon response: a new perspective on a familiar theme. Pathog Immun. 2018;3(1):126-48.

24 Fatica A, Bozzoni I. Long non-coding RNAs: new players in cell differentiation and development. Nat Rev Genet. 2014 Jan;15(1):7-21.

25 Shore AN, Rosen JM. Regulation of mammary epithelial cell homeostasis by $\operatorname{lncRNAs}$. Int J Biochem Cell Biol. 2014 Sep;54:318-30.

26 Ribeiro DM, Zanzoni A, Cipriano A, Delli Ponti R, Spinelli L, Ballarino M, et al. Protein complex scaffolding predicted as a prevalent function of long non-coding RNAs. Nucleic Acids Res. 2018 Jan 25;46(2):917-28.

27 Carnero E, Barriocanal M, Segura V, Guruceaga E, Prior C, Börner K, et al. Type I interferon regulates the expression of long noncoding RNAs. Front Immunol. 2014;5:548.

28 Fortes P, Morris KV. Long noncoding RNAs in viral infections. Virus Res. 2016 Jan 2;212: $1-11$

29 Peng X, Gralinski L, Armour CD, Ferris MT, Thomas MJ, Proll S, et al. Unique signatures of long noncoding RNA expression in response to virus infection and altered innate immune signaling. mBio. 2010 Oct 26;1(5): e00206-10.

30 Ren G-J, Fan X-C, Liu T-L, Wang S-S, Zhao G-H. Genome-wide analysis of differentially expressed profiles of mRNAs, lncRNAs and circRNAs during cryptosporidium baileyi infection. BMC Genomics. 2018 May 10;19(1): 356.

31 Wang $\mathrm{P}, \mathrm{Xu}$ J, Wang Y, Cao X. An interferonindependent lncRNA promotes viral replication by modulating cellular metabolism. Science. 2017 Nov 24;358(6366):1051-5.
32 Qian X, Xu C, Zhao P, Qi Z. Long non-coding RNA GAS5 inhibited hepatitis C virus replication by binding viral NS3 protein. Virology. 2016 May;492:155-65.

33 Ouyang J, Zhu X, Chen Y, Wei H, Chen Q, Chi X, et al. NRAV, a long noncoding RNA, modulates antiviral responses through suppression of interferon-stimulated gene transcription. Cell Host Microbe. 2014 Nov 12; 16(5):616-26.

34 Chai W, Li J, Shangguan Q, Liu Q, Li X, Qi D, et al. Lnc-ISG20 inhibits influenza a virus replication by enhancing ISG20 expression. J Virol. 2018 Aug 15;92(16):e00539-18.

35 More S, Zhu Z, Lin K, Huang C, Pushparaj S, Liang $Y$, et al. Long non-coding RNA PSMB8AS1 regulates influenza virus replication. RNA Biol. 2019 Mar;16(3):340-53.

$36 \mathrm{Hu}$ B, Huo Y, Yang L, Chen G, Luo M, Yang $J$, et al. ZIKV infection effects changes in gene splicing, isoform composition and lncRNA expression in human neural progenitor cells. Virol J. 2017 Nov 7;14(1):217.

37 Etebari K, Hegde S, Saldaña MA, Widen SG, Wood TG, Asgari S, et al. Global transcriptome analysis of Aedes aegypti mosquitoes in response to Zika virus infection. mSphere. 2017 Nov-Dec;2(6):e00456-17.

38 Frumence E, Roche M, Krejbich-Trotot P, ElKalamouni C, Nativel B, Rondeau P, et al. The South Pacific epidemic strain of Zika virus replicates efficiently in human epithelial A549 cells leading to IFN- $\beta$ production and apoptosis induction. Virology. 2016 Jun;493:21726.

39 Li C, Deng YQ, Wang S, Ma F, Aliyari R, Huang XY, et al. 25-Hydroxycholesterol protects host against Zika virus infection and its associated microcephaly in a mouse model. Immunity. 2017 Mar 21;46(3):446-56.

40 Li Y, Wu S, Pu J, Huang X, Zhang P. Dengue virus up-regulates expression of notch ligands Dll1 and Dll4 through interferon- $\beta$ signalling pathway. Immunology. 2015 Jan;144(1):12738.

41 Zhang P, Li Y, Xia J, He J, Pu J, Xie J, et al. IPS1 plays an essential role in dsRNA-induced stress granule formation by interacting with PKR and promoting its activation. J Cell Sci. 2014 Jun 1;127(Pt 11):2471-82.

42 Wang Y, Chen X, Xie J, Zhou S, Huang Y, Li $\mathrm{Y}-\mathrm{P}$, et al. RNA helicase A is an important host factor involved in dengue virus replication. J Virol. 2019 Feb 15;93(4):e01306-18.

43 Zhou S, Yang C, Zhao F, Huang Y, Lin Y, Huang C, et al. Double-stranded RNA deaminase ADAR1 promotes the Zika virus replication by inhibiting the activation of protein kinase PKR. J Biol Chem. 2019 Nov 29; 294(48):18168-80.

44 Wang Y, Chen X, Xie J, Zhou S, Huang Y, Li $\mathrm{Y}$, et al. RNA helicase A is an important host factor involved in dengue virus replication. J Virol. 2019;93(4):e01306-18. 
45 Ulitsky I, Bartel DP. lincRNAs: genomics, evolution, and mechanisms. Cell. 2013 Jul 3; 154(1):26-46.

46 Ranzani V, Rossetti G, Panzeri I, Arrigoni A, Bonnal RJ, Curti S, et al. The long intergenic noncoding RNA landscape of human lymphocytes highlights the regulation of $\mathrm{T}$ cell differentiation by linc-MAF-4. Nat Immunol. 2015 Mar; 16(3):318-25.

47 Son KN, Liang Z, Lipton HL. Double-stranded RNA is detected by immunofluorescence analysis in RNA and DNA virus infections, including those by negative-stranded RNA viruses. J Virol. 2015 Sep;89(18):9383-92.
48 Ma F, Li B, Yu Y, Iyer SS, Sun M, Cheng G. Positive feedback regulation of type I interferon by the interferon-stimulated gene STING. EMBO Rep. 2015 Feb;16(2):202-12.

$49 \mathrm{Ma} \mathrm{H}$, Han P, Ye W, Chen H, Zheng X, Cheng $\mathrm{L}$, et al. The long noncoding RNA NEAT1 exerts antihantaviral effects by acting as positive feedback for RIG-I signaling. J Virol. 2017 May 1;91(9):e02250-16.

50 Jiang M, Zhang S, Yang Z, Lin H, Zhu J, Liu $L$, et al. Self-recognition of an inducible host lncRNA by RIG-I feedback restricts innate immune response. Cell. 2018 May 3;173(4): 906-e13.
51 Li X, Guo G, Lu M, Chai W, Li Y, Tong X, et al. Long noncoding RNA Lnc-MxA inhibits beta interferon transcription by forming RNA-DNA triplexes at its promoter. J Virol. 2019 Nov 1;93(21):e00786-19.

52 Ngono AE, Shresta S. Immune response to dengue and Zika. Annu Rev Immunol. 2018 Apr 26;36:279-308.

53 Quinodoz S, Guttman M. Long noncoding RNAs: an emerging link between gene regulation and nuclear organization. Trends Cell Biol. 2014 Nov;24(11):651-63. 